\title{
The post-AGB evolution of AGB mass loss variations
}

\author{
R. Meijerink ${ }^{1}$, G. Mellema ${ }^{1}$, and Y. Simis ${ }^{1,2}$ \\ 1 Sterrewacht Leiden, PO Box 9513, 2300 RA, Leiden, The Netherlands \\ e-mail: mellema@strw.leidenuniv.nl;simis@strw.leidenuniv.nl \\ 2 Astrophysikalisches Institut Potsdam, An der Sternwarte 16, 14482 Potsdam, Germany
}

Received 11 December 2002 / Accepted 28 April 2003

\begin{abstract}
We present new numerical hydrodynamical modelling of the evolution of Asymptotic Giant Branch (AGB) mass loss fluctuations during the post-AGB/Planetary Nebula phase. These models show that after ionization, the observable effects of the mass loss fluctuations disappear in a few thousand years, consistent with the fact that only few PNe have been found to be surrounded by "rings". We derive the observational characteristics of these rings, and compare them to reported observations of the rings around NGC 6543, finding a good match of emission properties and line shapes. We predict small variations in the observable electron temperatures.
\end{abstract}

Key words. planetary nebulae: general - ISM: bubbles - stars: AGB and post-AGB - hydrodynamics

\section{Introduction}

High resolution imaging with the Hubble Space Telescope HST has revealed the presence of so-called "rings" around Planetary Nebulae (PNe) and proto-PNe. Examples can be found around the proto-PNe CRL 2688 (Sahai et al. 1998, Egg Nebula), IRAS 17441-2411 (Su et al. 1998), IRAS 17150-3224 (Kwok et al. 1998), IRAS 16594-4656, IRAS 20028+3910 (Hrivnak et al. 2001), and the PNe NGC 7027, (where they show up in scattered light), NGC 6543 and Hb 5 (Terzian \& Hajian 2000). Inspection of HST archival images of NGC 3918 also reveal ring-like structures in its halo, confirmed by ground-based data (Corradi et al. 2003). Similar rings are observed around one AGB star, the extreme carbon star IRC+10216 (Mauron \& Huggins 2000).

The name "rings" is somewhat of a misnomer since these are three-dimensional structures. However, the term is now commonly used and we will adher to this convention. Although never completely closed, the rings show an overall spherical appearance, and seem to be associated with a time when the mass loss from the AGB star was roughly spherical. Interestingly, they tend to sit right around the aspherical nebular structures, suggesting they are connected to the phase just before the onset of asphericity in the system.

Their origin has been attributed to three different processes. Mastrodemos \& Morris (1999) showed that binary interaction can produce ring-like structures, both in and perpendicular to the orbital plane. In this case the rings are actually the spiral waves produced by the presence of the companion star. García-Segura et al. (2001) reproduce the ring features by

Send offprint requests to: R. Meijerink, e-mail: meijerin@strw.leidenuniv.nl invoking a magnetic cycle, similar to that in the Sun, for the AGB star. The period is then set by the period of this magnetic activity cycle, which is a parameter in their model. Frank (private comm.) has pointed out that any magnetic dynamo cycle in AGB stars would have a much shorter period than the one corresponding to the rings. The third model comes out of a study of the dust-driven winds of AGB stars. Simis et al. (2001) showed that intricate coupling between dust and gas, leads to cycles in which predominantly either large or small grains are produced, which then produce periodic variations in the wind density and velocity. The period depends on the sound travel time of the subsonic wind region, and is found to lie close to the observed values for the rings.

In case the rings are ionized it is possible to use plasma diagnostics to derive the temperature and density in the rings. To date this has not been done in detail. Balick et al. (2001) estimated some properties of the rings around NGC 6543 on the basis of HST images and limited spectroscopic data. Hyung et al. (2001) used HST images taken with WFPC2 in narrow band filters near the lines of [O III] $5007 \AA$ and $4363 \AA$ to find the electron temperature in the rings of the same object. Although Balick et al. (2001) found no indication for unusual electron temperatures in the rings, Hyung et al. (2001) claim to find substantially higher electron temperatures, indicating the presence of shocks. Both methods have their limitations, and the result remains inconclusive. Balick et al. (2001) derived density variations from $\mathrm{H} \alpha$ emission variations, and found them to be consistent with individual shells with negligible amounts of material between them.

Both Bryce et al. (1992) and Balick et al. (2001) report that emission lines in the rings area of NGC 6543 are unusually broad, with FWHM of around $30 \mathrm{~km} \mathrm{~s}^{-1}$, for which they could 
offer no explanation. Hyung et al. (2001) presented a simple model for explaining the putative higher electron temperatures, as well as the broadened emission lines. Following the evolution of sinusoidal density and velocity variations using a numerical hydrodynamic model including the effects of ionization, they could reproduce temperatures and broadened lines. However, the model only included the rings, not the core nebula, nor did it follow the full evolution of the rings, and in order to keep the velocity field under control, unreasonably high velocities had to be used.

Studying the evolution of the rings from the AGB to the PN phase is interesting for several reasons. Firstly, one may be able to find differences between the three proposed models. Secondly, it can help us understand whether the limited number of PNe found to have rings is intrinsic, e.g. because ionization changes the properties of the rings, or rather due to lack of high resolution observations. Also, it will provide insight in the connection between the AGB mass loss variations and the ionized PN rings.

In this paper we study the evolution of mass loss variations on the AGB through the proto-PN phase to the PN phase. For this we will use an improved version of the model of Simis et al. (2001) to produce the AGB mass loss variations, and an improved version of the model used in Mellema (1994) to follow their evolution during the post-AGB phase. Although we pick one model for the origin of the mass loss variations, we will show that most of the results have relevance for the other models too.

The layout of the paper is as follows. In Sect. 2 we describe the numerical methods used to produce the mass loss variations and to study their fate from the AGB through the post-AGB phase. Section 3 presents the results of a typical simulation, for which we derive observables in Sect. 4. In Sect. 5 we discuss the results and how they increase our understanding of the origin and evolution of the rings. Our conclusions are summed up in Sect. 6.

\section{Two models}

In order to model the evolution of AGB mass loss variations we first need to produce them. To keep the model as physical as possible, we produce the mass loss fluctuations using a slightly improved version of the two-fluid dust-gas code described by Simis et al. (2001). These are then used as input for a gas-only hydrodynamics code which can treat the detailed ionization, heating and cooling processes in step with the evolution of the star. We will now describe these codes in some more detail.

\subsection{A dust-driven AGB wind model}

We provide here a short description of the two-fluid hydrodynamics code which we use to model the AGB. For a more detailed description of the code and the implementation of the two-fluid flow in particular, the reader is referred to Simis et al. (2001, 2003). The new aspect of this code is the fact that it combines time-dependent hydrodynamics with two-fluid flow and a self consistent description of the dust (including nucleation and growth). It turns out that the interaction of these physical mechanisms is critical for the formation of the shells, which are a result of alternating low- and high-mass loss episodes of outflow. The mass loss rate is low when the momentum transfer from grains to gas is inefficient. This is the case when the gas has a relatively low velocity while the dust grains move relatively fast (i.e. they have a high drift velocity). This results in a rapid passage of the grains through the zone in which grain growth is efficient, and hence in somewhat smaller grains. As a consequence, the drag force exerted on the gas is relatively weak. This leads to a decrease of the gas density and hence an even larger drift velocity, because the frequency of gas-grain collisions decreases. So, during the low mass-loss phase the average grain size gradually decreases whereas its drift speed increases. In a phase of high mass loss, the rate of momentum transfer from the radiatively accelerated grains to the gas is high. This results in a large velocity of the gas and a low drift velocity. Because the grains move relatively slow through the gas they have sufficient time to grow. The average grain size during this phase is relatively large, which causes an efficient momentum transfer. This enables a high gas density, a gradual growth of the grains and a further decrease of their drift speed. The transition between the two phases is triggered by the sudden increase of the drag force at the end of the low mass loss phase, which is a result of the quadratic appearance of the (increasing) drift velocity in the drag force. The sudden increase of the drag force leads to an increasing momentum transfer per collision and it prevents the ongoing decrease of the collision rate that was a result of the gradually decreasing grain size. This starts the high mass loss phase. Curiously, it also triggers the onset of the next low mass loss phase: due to the suddenness of the transition, an outward shock develops. At the same time, a rarefaction wave moves towards the stellar surface. This wave will dilute the gas, thereby again creating a condition for inefficient grain growth, small grains, low collisional cross sections and hence a low mass loss rate. Hence, the characteristic time scale for the mass loss fluctuations is the time used by the rarefaction wave to cross the dust forming part of the subsonic region of the outflow.

The code is a time dependent, Eulerian, explicit hydrodynamics code, based on the FCT/LCD algorithm assuming spherical symmetry (Boris 1976; Icke 1991). Physical processes taken into account in the code involve (equilibrium) gas chemistry, nucleation and growth of grains (Gail et al. 1984; Gail \& Sedlmayr 1988; Dorfi \& Höfner 1991), semi-analytical radiative transfer (Fleischer et al. 1992) and the behaviour of grains in collision with gas particles. The temperature is calculated assuming local radiative equilibrium, following Lucy (1976). Grain-gas collision is implemented to provide a realistic description of the momentum transfer between the two fluids. This enables a full two-fluid description, i.e. no assumptions about the motion of the grains relative to the gas need to be made. The extra degree of freedom introduced thereby turns out to be essential for the formation of quasi periodic shells: when comparing two otherwise identical model calculations, the (two-fluid) model in which grains can drift with respect to the gas does produce the shells, whereas the single fluid model, i.e. the model in which grains are always assumed to have the same velocity as the gas, has a more or less stationary outflow. 


\subsection{A post-AGB model}

The numerical method we use to follow the subsequent evolution in the post-AGB phase, descends from the method used by Mellema (1994). This code solves the hydrodynamic equations in one-dimension, using the spherical radial coordinate. The solver of choice is the "Roe solver" (Roe 1981; Eulderink \& Mellema 1995), a second-order accurate, approximate Riemann solver.

The atomic physics is treated according to the approach described in Mellema \& Lundqvist (2002) (DORIC), but with the addition of photo-ionization from an evolving source. This means that we follow the time-dependent ionization of all ions of $\mathrm{H}, \mathrm{He}, \mathrm{C}, \mathrm{N}, \mathrm{O}$, and $\mathrm{Ne}$, taking into account collisional ionization, photo-ionization, radiative and dielectronic recombination and charge exchange with hydrogen. The local density and temperature of the ions is used to calculate the local nonequilibrium cooling rate. The heating rate is given by photoionization heating of $\mathrm{H}$ and $\mathrm{He}$.

The transfer of ionizing radiation is treated according to the method described in Frank \& Mellema (1994) and Mellema et al. (1998), i.e. using the three frequencies corresponding to the ionization thresholds of $\mathrm{H}^{0}, \mathrm{He}^{0}$, and $\mathrm{He}^{+}$, but taking the frequency dependence of the cross sections into account when calculating the integrals over the spectral energy distribution. At all times, the star is assumed to radiate as a black body with the effective temperature given by the stellar evolution model used (see below).

The outer boundary is an outflow condition, the inner boundary condition is provided by the evolving stellar wind in the post-AGB phase.

\subsubsection{The post-AGB wind}

During the post-AGB phase a star can reach temperatures over $10^{5} \mathrm{~K}$. The bulk of the radiation is emitted in the UV, where the outer layers have an abundance of absorption lines. Castor et al. (1975) found that for hot stars, absorption lines dominate in transferring momentum from the radiation to the gas, and the consequent stellar wind is therefore called "line-driven". The theory of line-driven winds is well developed and tested. Here we use the analytical approximation derived by Kudritzki et al. (1989), which for a given effective temperature and luminosity gives the stellar wind mass loss rate and terminal velocity.

The mass loss rate of a line-driven wind is proportional to the luminosity and the number of absorbing lines. The acceleration of the atoms is proportional to a so called force multiplier, which is approximated by a simple function:

$M(t)=k t^{-\alpha}\left(10^{-11} n_{\mathrm{e}} / W\right)^{\delta}$,

where $t$ is a dimensionless optical depth parameter, $k, \alpha$ and $\delta$ force multiplier parameters and $W$ the dilution factor. The acceleration also depends on the velocity field in the wind as a function of radius. This velocity field is given by:

$v(r)=v_{\infty}\left(1-\frac{R_{*}}{r}\right)^{-\beta}$.
Apart from the stellar luminosity and effective temperature, the model also needs values for these force multiplier parameters, $k, \alpha, \beta$ and $\delta$, as well as for the stellar mass, the helium abundance, and the average number of electrons produced by helium.

The luminosity, mass and effective temperature are taken from Blöcker (1995), who presented a compilation of evolutionary tracks for post-AGB masses in the range 0.53 to $0.94 M_{\odot}$. Three of these tracks were originally calculated by Schönberner (1983). The force multiplier parameters we take from the compilation in Lamers \& Cassinelli (1999, Table 8.2).

With these parameters the wind depends strongly on the stellar effective temperature. When the star just enters the postAGB phase, its effective temperature is low, and the mass loss rate and wind velocity are low too. As the effective temperature goes up, the mass loss rate increases, levelling off to a roughly constant rate from about $10000 \mathrm{~K}$. At this point the number of effective lines reaches a maximum. When nuclear burning stops, the luminosity of the star starts to drop and the mass loss rate goes down, but the velocity of the wind keeps rising. Figure 1 shows the evolution of the wind as a function of stellar effective temperature for four different evolutionary tracks.

\section{Simulations}

We performed five simulations for different initial density distributions and stellar masses. In this section we describe the results of one and refer to the results of the other simulations only when they help us understand certain features of behaviour.

\subsection{The initial density distribution}

In Sect. 2.1 the mechanism of the mass loss variations during the AGB was discussed. From the two-fluid dust-gas simulations of Simis et al. (2001) we took one result as the input for our simulation. These mass loss and velocities variations were calculated using the properties of IRC+10216 (Winters et al. 1994): a stellar mass of $0.7 M_{\odot}$, an effective temperature of $1.98 \times 10^{3} \mathrm{~K}$, luminosity $2.4 \times 10^{4} L_{\odot}$, and a carbon/oxygen ratio of 1.4 .

Our main aim is to follow the post-AGB evolution of the type of variations in density and velocity which are being produced by the two-fluid calculations. We therefore scale the outcome of the two-fluid simulation to obtain a more typical AGB wind with an average mass loss rate of $2.5 \times$ $10^{-5} M_{\odot} \mathrm{yr}^{-1}$, and an average velocity of $10 \mathrm{~km} \mathrm{~s}^{-1}$. This means that the AGB and post-AGB simulations are no longer physically connected and the parameters used for the AGB star listed above are no longer relevant for the results. What we are effectively doing is using the two-fluid calculations to generate a spectrum of density and velocity variations which at least has some physical basis (rather than assuming sinusoidal variations), and apply this to our AGB wind. This is also motivated by the fact that the quantitative outcome of the two-fluid simulation is quantitatively uncertain since the model does not include effects such as pulsation, evolution of the AGB star, etc.

The computational grid of the dust-gas simulations extended to a radius of only $1.8 \times 10^{16} \mathrm{~cm}(1200 \mathrm{AU})$ and to be 

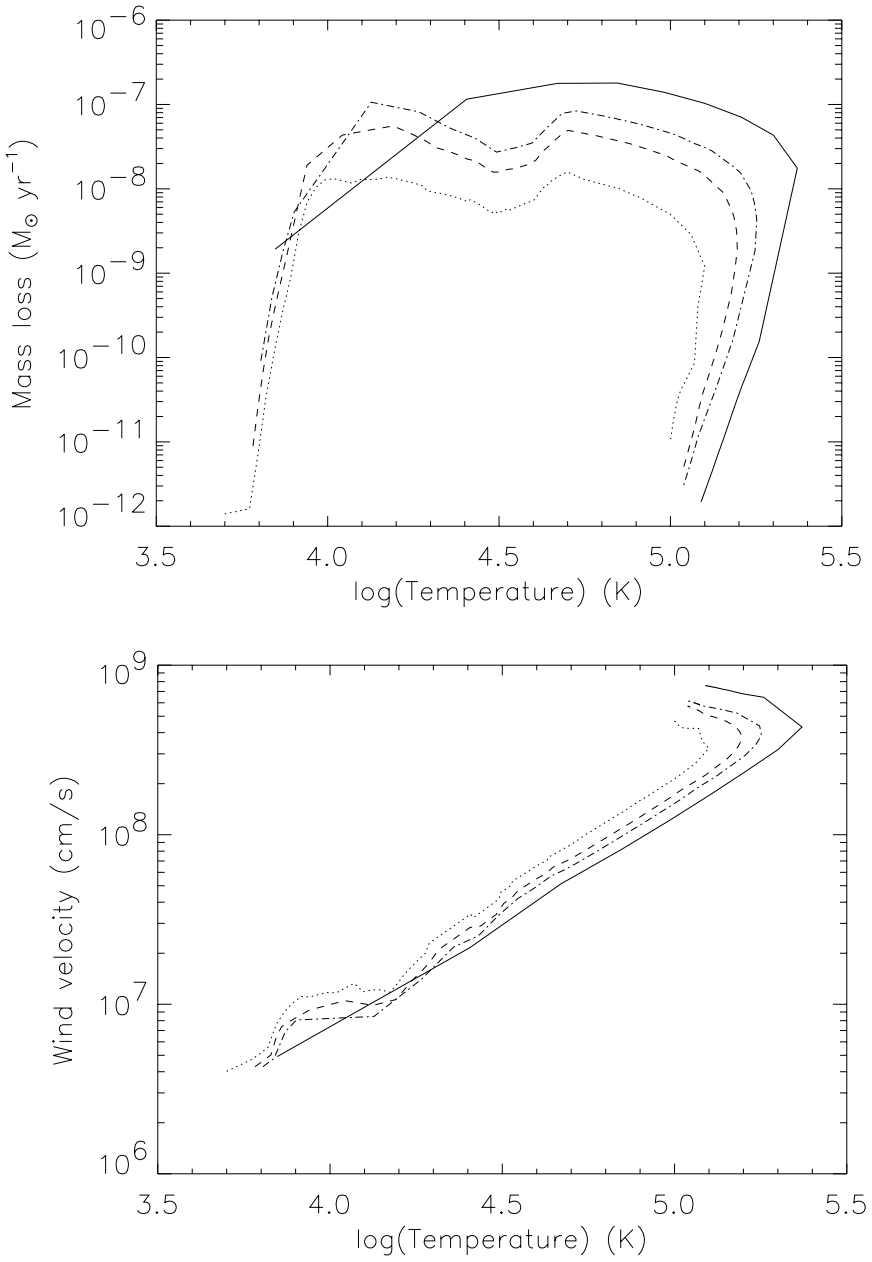

Fig. 1. The mass loss of the star and the velocity of the wind as a function of effective temperature, for post-AGB masses of $0.565 M_{\odot}$ (dotted), $0.605 M_{\odot}$ (dashed), $0.625 M_{\odot}$ (dashed dotted) and $0.696 M_{\odot}$ (solid).

able to follow the long term (post-AGB) evolution we first had to fill a larger space with mass loss and velocitiy fluctuations. For this we extracted the density and velocity as a function of time at a radius of $5.0 \times 10^{15} \mathrm{~cm}(333 \mathrm{AU})$, and used this as an inflow condition for a period of 31000 years, by which time the fluctuations fill most of the grid. The temperature at the inner boundary is taken from the two-fluid calculation, and we extrapolate outward using a power law, $T \propto r^{-2(\gamma-1)}$, with an imposed minimum value of $10 \mathrm{~K}$. The actual choice for the temperature profile is not very important for the dynamics, since the gas is flowing out supersonically.

As the fluctuations move out, the distances between minima in density become larger. This is due to the merging of shells, since these do not have the same velocity. Figure 2 shows the structure of the AGB wind 31000 years after we introduced the variable wind. One sees that the spacing of the rings increases with distance from the star. When this density pattern is projected on the sky the highest frequency structures disappear. As a consequence it is easier to distinguish the shells.
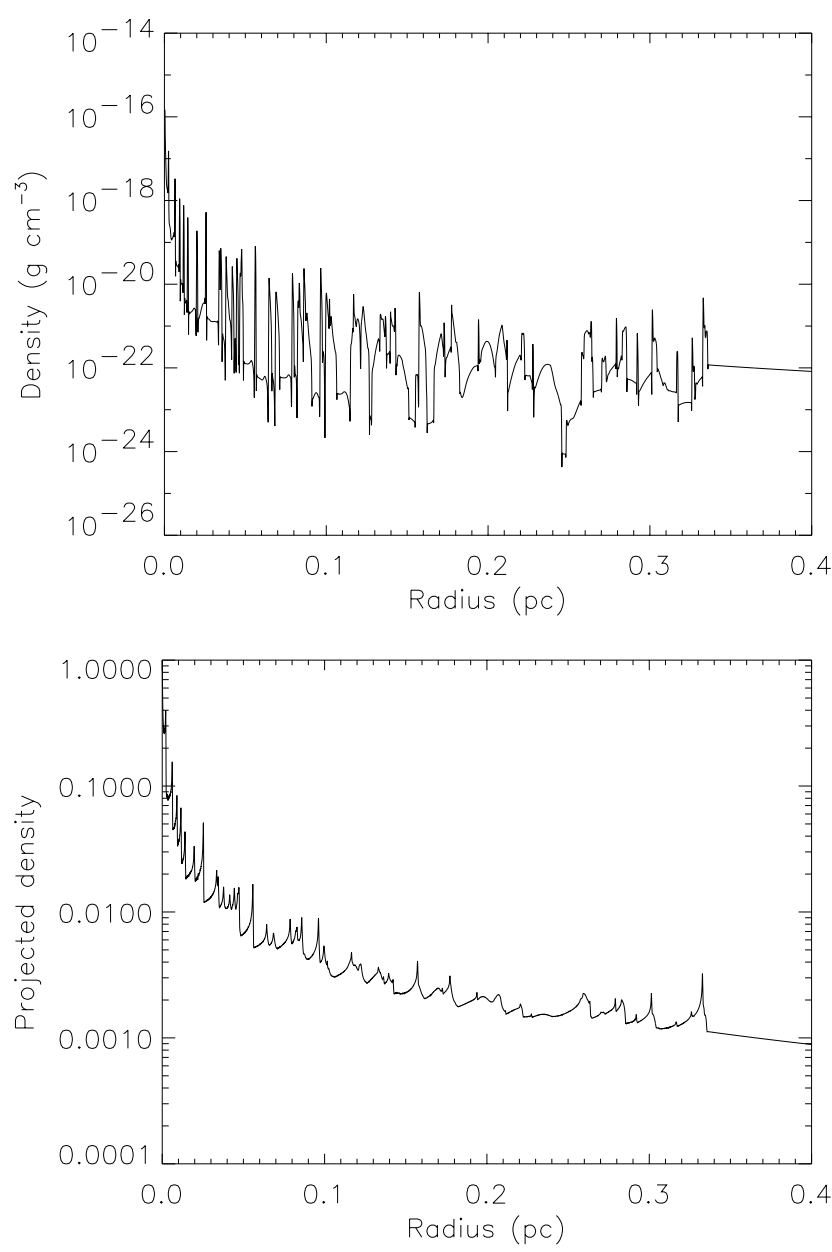

Fig. 2. Density profile as a function of radius (top) and projected on the sky (bottom).

\subsection{The dynamical evolution of the rings}

Our standard model is for a star with a Zero Age Main Sequence (ZAMS) mass of $1.0 M_{\odot}$ and post-AGB mass of $0.565 M_{\odot}$. We call $t=0$ the start of this simulation, which corresponds to the start of the post-AGB evolution. The simulation follows the evolution of the circumstellar material over a time span of 15000 years and is performed on a grid of 6000 points. The post-AGB wind first collides with the slow AGB wind at a distance of $5 \times 10^{15} \mathrm{~cm}$, the inner edge of the grid lies at $10^{14} \mathrm{~cm}(6.6 \mathrm{AU})$.

In Fig. 3 the result after 400 years is shown. The effective temperature of the star is about $6000 \mathrm{~K}$. There are enough energetic photons to ionize the fast wind. Because the velocity of the fast wind is $50 \mathrm{~km} \mathrm{~s}^{-1}$ and the mass loss rate of the star $1.6 \times 10^{-12} M_{\odot} \mathrm{yr}^{-1}$, the temperature in the shock, moving into the fast wind (the inner shock), is not that high, $\sim 3000 \mathrm{~K}$. The pressure in the inner shock is actually lower than in the slow wind. As a consequence, the slow wind dissipates slightly inwards. The ionization front lies at the interaction between the fast and the slow wind.

Six hundred years later (Fig. 4), the effective temperature is $8200 \mathrm{~K}$, and the mass loss rate went up by three orders of magnitude to $\sim 10^{-9} M_{\odot} \mathrm{yr}^{-1}$, with a velocity to somewhat over $100 \mathrm{~km} \mathrm{~s}^{-1}$. As a consequence, the temperature in the hot 

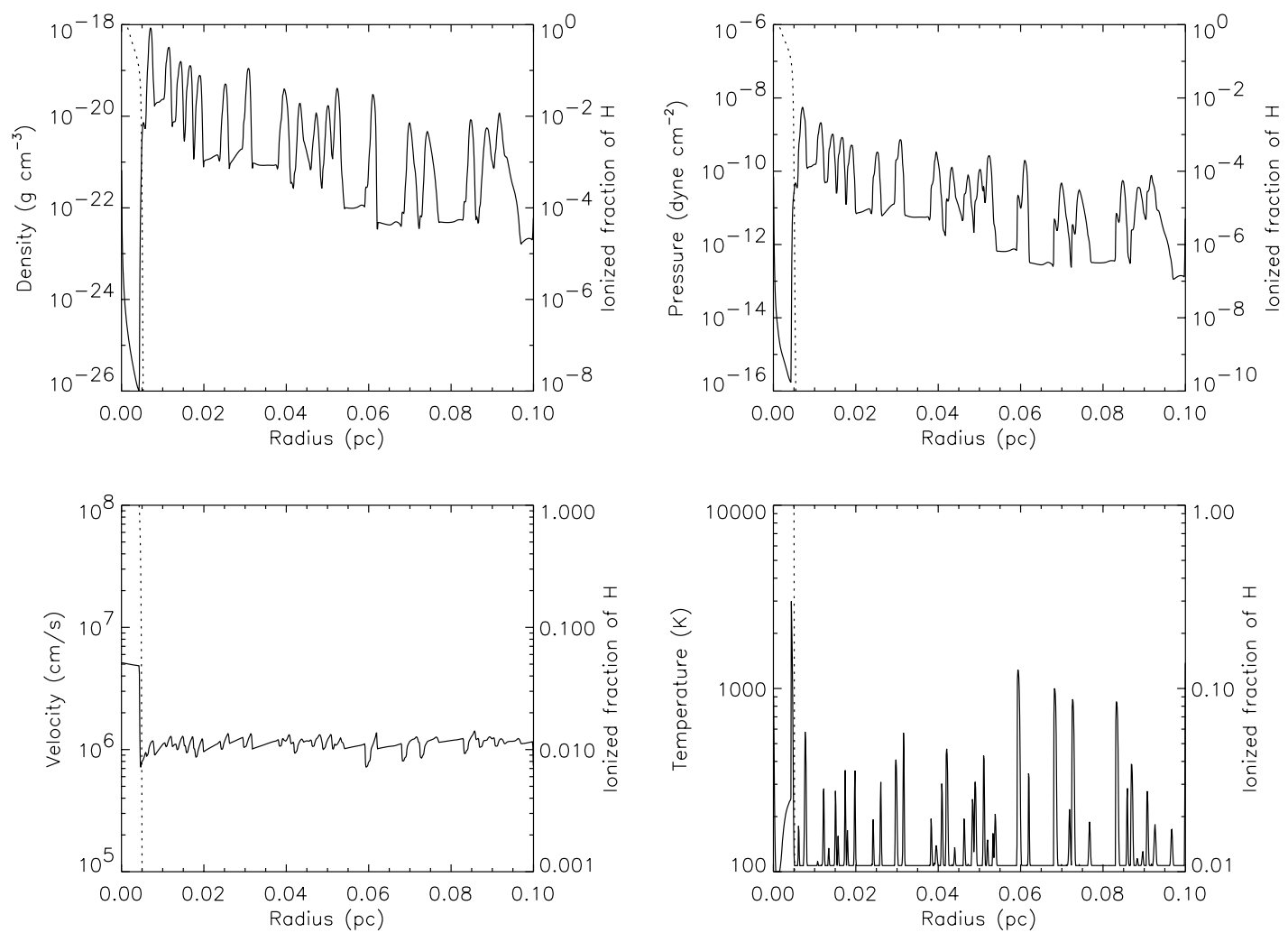

Fig. 3. Result after 400 years: density (top left), pressure (top right), velocity (bottom left) and temperature (bottom right). The dotted line shows the ionized fraction of hydrogen.

bubble is well over $10^{5} \mathrm{~K}$. The pressure is now the same as in the slow wind. One can also see the ionization front moving into the slow wind. The temperature here is about $2500 \mathrm{~K}$. The gas in front of the ionization front is not shocked yet and has a temperature around $100 \mathrm{~K}$. One also sees that the shells merge while they move outward. At the place where the shells interact the temperature rises to a few hundred degrees.

The ionization front has moved further out to $0.15 \mathrm{pc}$, 2100 years later (see Fig. 5), when the star's effective temperature has risen to $20000 \mathrm{~K}$. The part of the wind that is ionized has almost no density variations. Since the pressure of the gas in the ionized region is higher, the gas in front of the ionization front is pushed outward. This gas piles up in a thin shocked shell and with a temperature somewhat lower than the ionized region. This is typical of a D-type ionization front, where the speed of the front is slow enough for the gas to react to the increased pressure of the ionized region. This configuration also produces the typical positive velocity gradient in the ionized material, found in many PNe.

Almost 3700 years after the end of the AGB (see Fig. 6) the star's effective temperature $(\sim 24000 \mathrm{~K})$ reaches the point that the ionization front breaks through. In a few hundred years the whole nebula gets ionized and goes from ionization bounded to density bounded. The temperature in the ionized part of the slow wind rises to about $7000 \mathrm{~K}$, and as a consequence the pressure also rises. The pressure being proportional to $\rho T$, follows the density profile and large pressure differences of an order of a magnitude occur. The gas tries to equalize this out, the effect of which can be seen in the velocity field. In the ionized region the velocity variations are over a shorter distance and have a larger amplitude than in the neutral region.

At 4500 years (see Fig. 7) the velocity variations have increased, and the velocity in the rings now range from 1 to $20 \mathrm{~km} \mathrm{~s}^{-1}$. These variations are much larger than in the period before ionization. To check the dependence on the initial velocity fluctuations, we did a simulation with the same initial density variations but with a constant velocity field. We found essentially the same velocity variations developing after ionization. This shows that the initial velocity variations are unimportant in this phase.

Considering the situation 3500 years later (see Fig. 8), we find that the density and velocity variations are still visible. These variations eventually equalize out, but even after 15000 years some traces of it can still be distinguished.

\section{Synthesized observations}

\subsection{Emission lines and temperature}

A radial density profile does not necessarily correspond to what would be observed. In order to compare to the observations, we calculated emissivities of certain lines, in [O III] $5007 \AA$, the line in which the rings in NGC 6543 are most easily observed, as well as the [O III] $4363 \AA$ line, whose ratio with the 5007 line gives us an "observed temperature". We calculated the line emissivity from the density, temperature, and $\mathrm{O}^{2+}$ concentration at every point. The emission is calculated conform Sect. 3.5 and Tables 3.4 and 3.8 of Osterbrock (1989). 

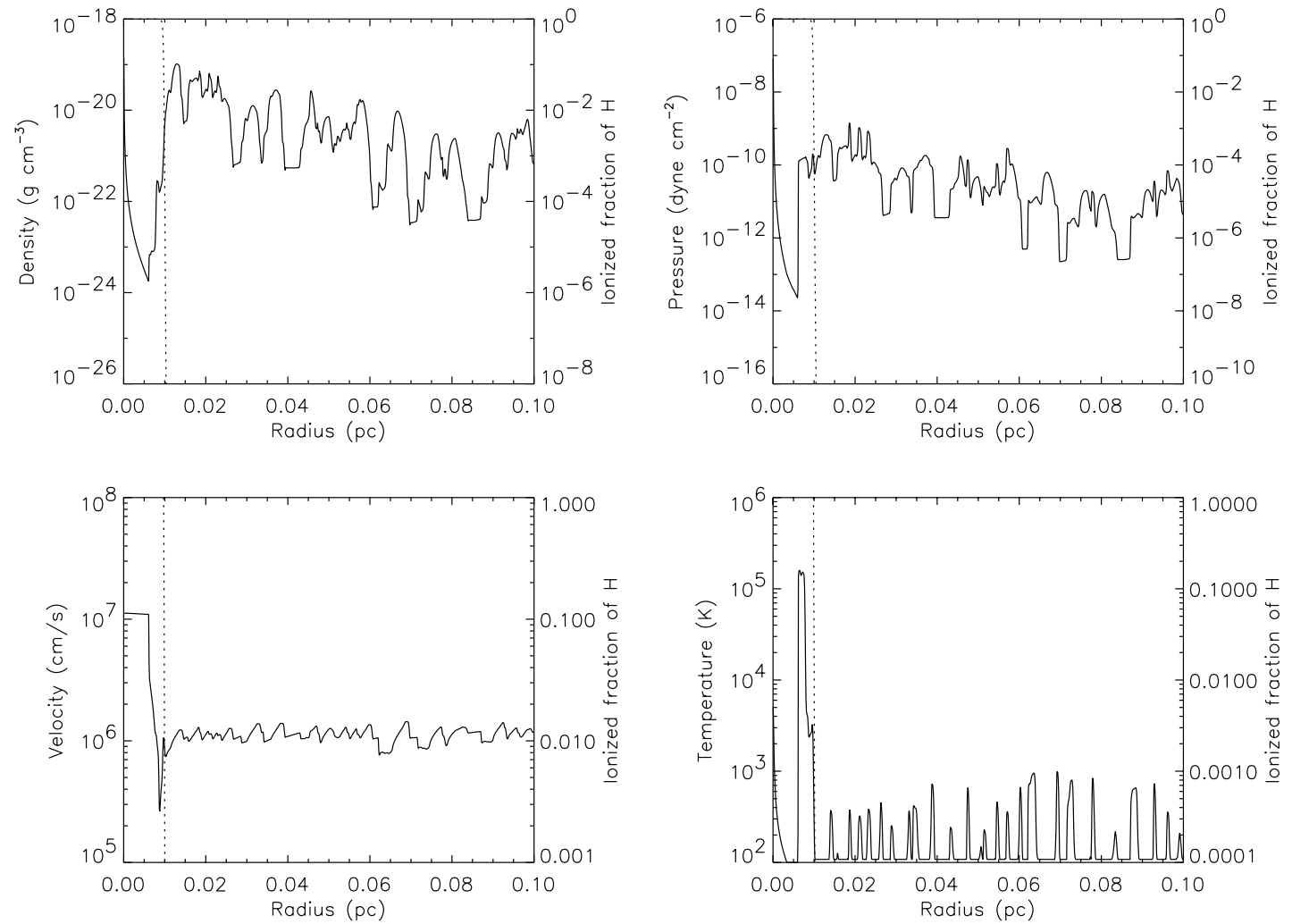

Fig. 4. Result after 900 years: density (top left), pressure (top right), velocity (bottom left) and temperature (bottom right). The dotted line shows the ionized fraction of hydrogen.
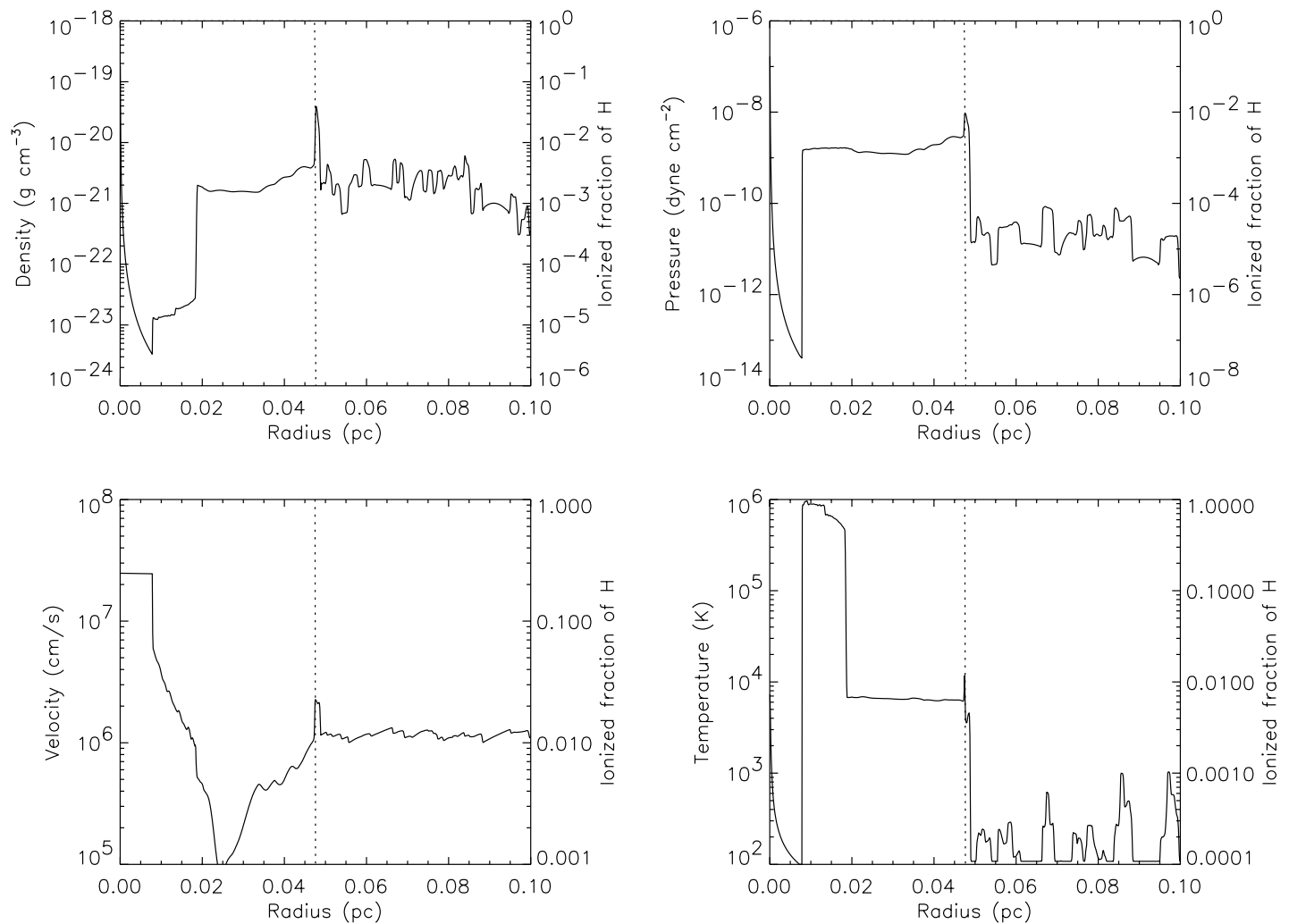

Fig. 5. Result after 3000 years: density (top left), pressure (top right), velocity (bottom left) and temperature (bottom right). The dotted line shows the ionized fraction of hydrogen. 

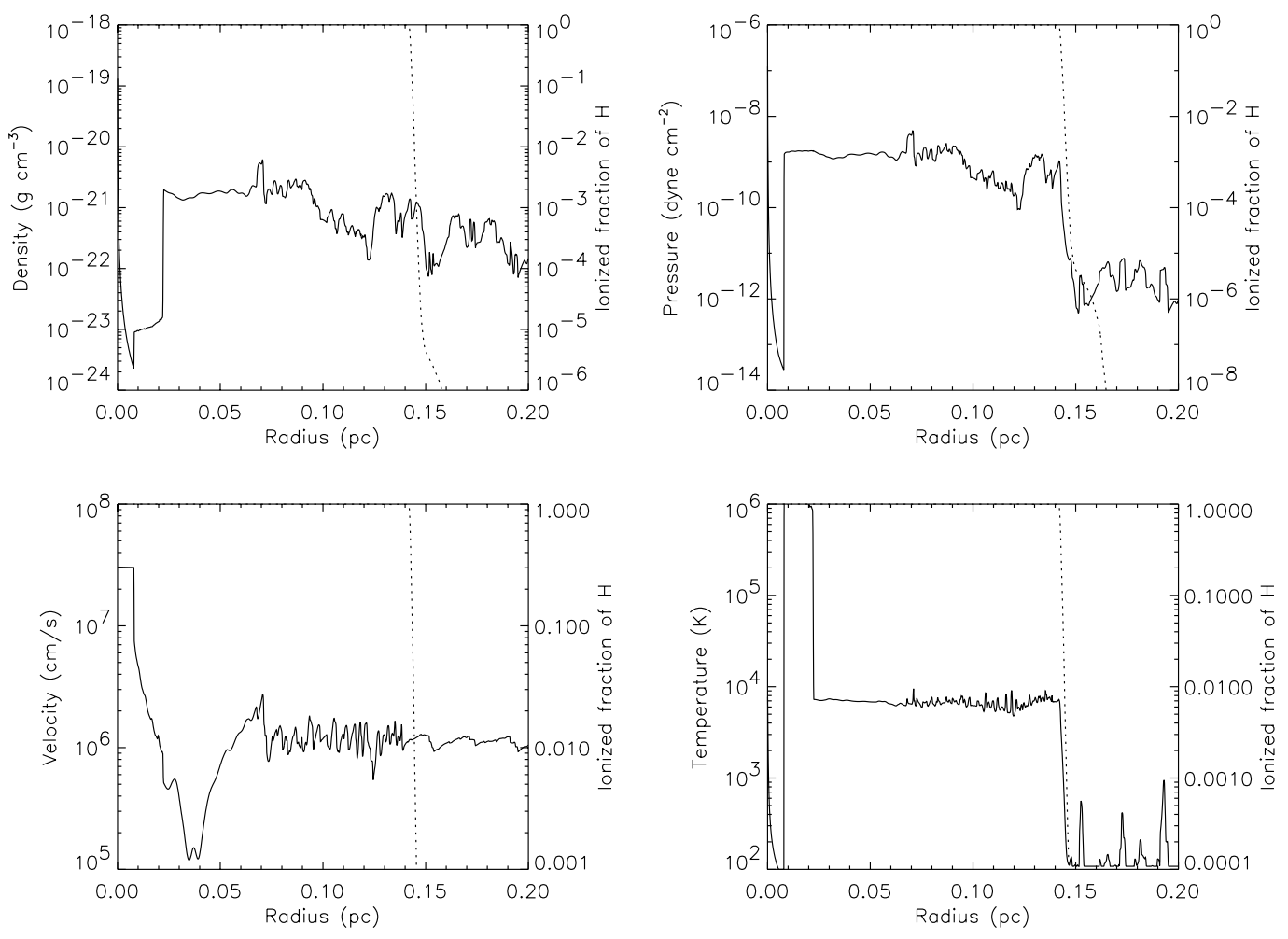

Fig. 6. Result after 3700 years: density (top left), pressure (top right), velocity (bottom left) and temperature (bottom right). The dotted line shows the ionized fraction of hydrogen.
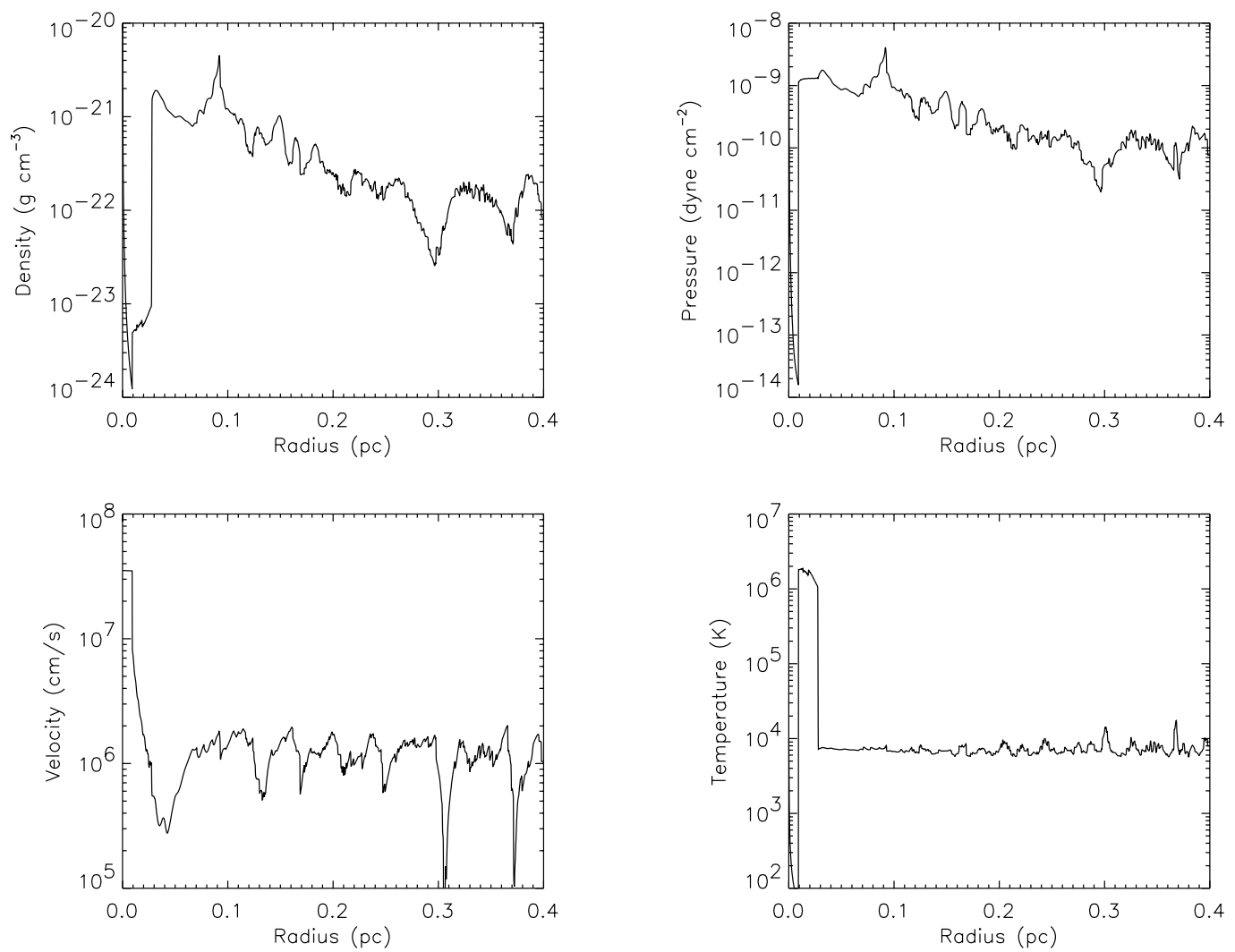

Fig. 7. Result after 4500 years: density (top left), pressure (top right), velocity (bottom left) and temperature (bottom right). All the hydrogen is ionized. 

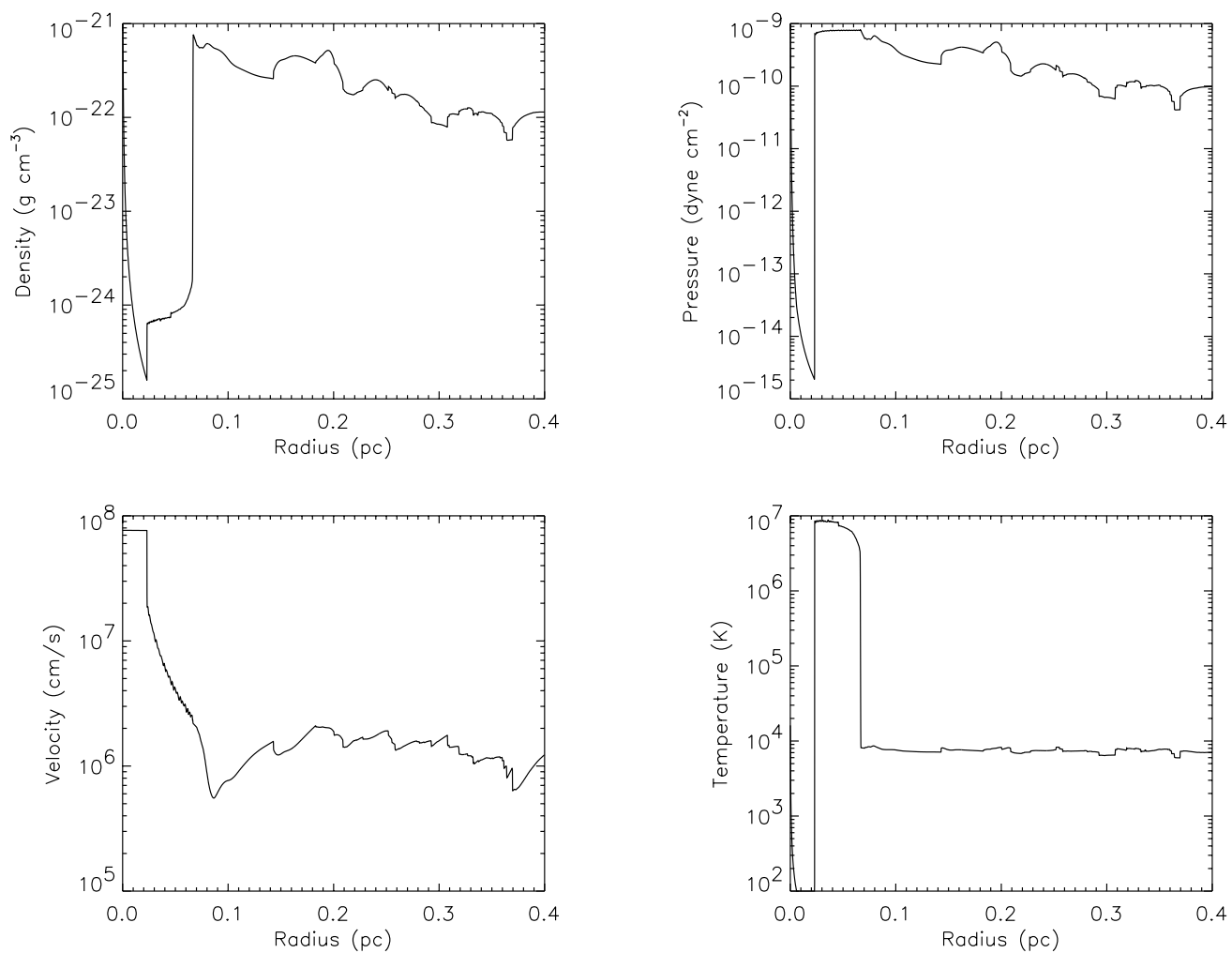

Fig. 8. Result after 8000 years: density (top left), pressure (top right), velocity (bottom left) and temperature (bottom right).

The radial [O III] $5007 \AA$ and $4363 \AA$ emissivities at $t=$ 4500 years are shown in Fig. 9, together with the density and temperature. One can see that the density profile does not correspond to the [O III] profiles. This is due to the temperature dependence of the [O III] line which gives more weight to points of higher temperature, especially for the $4363 \AA$ line.

To compare to observations, we projected the emissivities onto the sky, and convolved them with a Gaussian of FWHM corresponding to $0.1 \mathrm{arcsec}$ at the distance of NGC 6543 . We then took the ratio between the 5007 and $4363 \AA$ lines to determine the observed temperature. These projected emission and temperature profiles for the rings area are shown in Fig. 10.

The [O III] emission profile shows clear maxima and minima. The spacing between the maxima is about $0.02 \mathrm{pc}$. The dashed line shows the same in $\mathrm{H} \alpha$. Clearly the two have different radial dependencies. Figure 2 of Balick et al. (2001) shows how the rings are much more pronounced in the [O III] profile than in the $\mathrm{H} \alpha$ profile and how the $\mathrm{H} \alpha$ emission falls off more rapidly, something which our model at least qualitatively reproduces.

The observed temperature varies between $7500 \mathrm{~K}$ and $10000 \mathrm{~K}$, but most of this is due to a small outward gradient. Since the radial temperature profile does not show a temperature gradient, we must conclude that this is a projection effect. This is an interesting side-result, showing another way in which deriving electron temperatures from line ratios can be somewhat misleading. The true local variations are mostly of order $500 \mathrm{~K}$. The biggest variations in temperature are seen in the outer part of the nebula, where most shocks occur at this time.
One thousand years later (Fig. 11), the temperature variations have decreased substantially. The largest variations are still seen in the outer region, where the shells are merging. Due to this merging the spacings between maxima have become larger.

At $t=8000$ years almost no temperature variations are seen, the amplitude has dropped below a few hundred Kelvin (Fig. 12). Also in the density profile the rings have become less noticeable, although they can still be distinguished.

\subsection{Lineprofiles}

Combining the emissivities with the velocity information from the simulation, allows us to derive line shapes. We present line shapes at different positions along the rings in Fig. 13. Since the temperature of the gas is around $7500 \mathrm{~K}$, the lines are also thermally broadened which we simulated by convolving with a Gaussian of $6 \mathrm{~km} \mathrm{~s}^{-1}$ FWHM. The effects of seeing or slit width were not taken into account here.

One sees how the profiles are quite broad, up to $30 \mathrm{~km} \mathrm{~s}^{-1}$ FWHM. This is due to the fact that at a given position one observes several overlapping shells producing different velocity components. The profiles are clearly not Gaussian, so the effect that the emission comes from different shells is visible.

These line profiles match the reported observations quite well. Bryce et al. (1992) and Balick et al. (2001) describe (but do not show) wide $\left(\sim 30 \mathrm{~km} \mathrm{~s}^{-1}\right)$ line profiles in the region of the rings. The widths match our model line profiles well, the low $\mathrm{S} / \mathrm{N}$ of the spectroscopy of the faint rings makes further comparison difficult. 

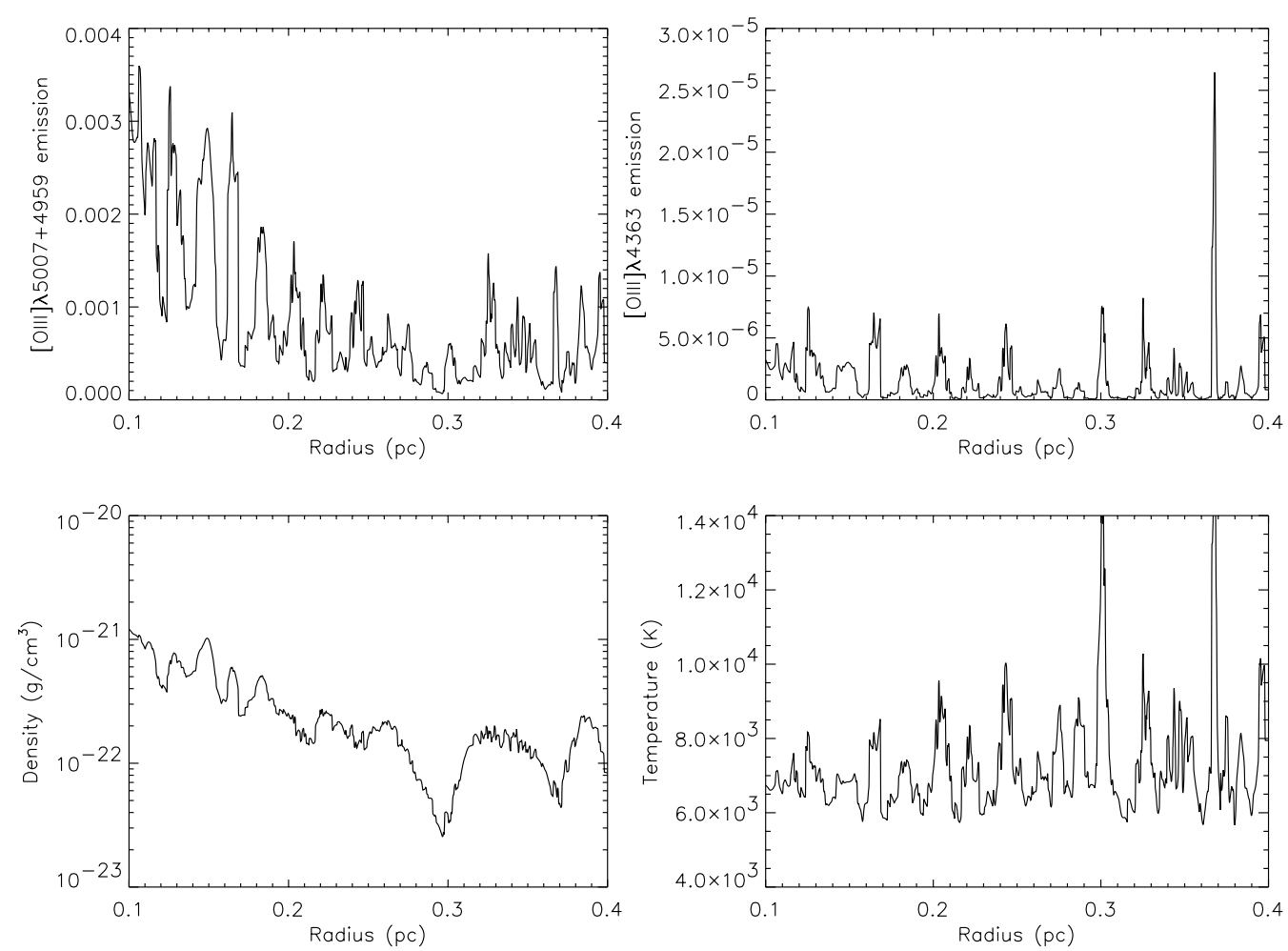

Fig. 9. Emission from [OIII] $\lambda 4959+\lambda 5007$ (top left), $\lambda 4363$ (top right) in arbitrary units, density profile (bottom left) and temperature profile (bottom right) at $t=4500$ years.

\section{Discussion}

The comparison with the observations of NGC 6543 in the previous section shows that our model matches a number of key observations:

1. the rings are clearly visible in the [O III] images;

2. the $\mathrm{H} \alpha$ and [O III] emissivities have slightly different radial profiles;

3. the line profiles are broadened to $\sim 30 \mathrm{~km} \mathrm{~s}^{-1}$.

This shows that our model gives a good description of the rings.

Comparing the spacing from our models with what is found in NGC 6543, we find a small descrepancy. Our spacing is $0.02 \mathrm{pc}$, about twice as large as in the observations (0.01$0.015 \mathrm{pc}$ ), assuming the distance to NGC 6543 is reliable. However, the exact spacing of the rings is influenced by many factors. First, the original spacing in the variable AGB mass loss depends on the density scale height at the base of the circumstellar envelope. Second, over time shells merge, increasing the spacing, and hence making it a function of time. A faster evolution will thus produce shorter distances between the shells.

The derived observable temperature (from the [O III]4363/5007 ratio) is found to vary somewhat, but not as much as claimed by Hyung et al. (2001), supporting the results of Balick et al. (2001) for an electron temperature comparable to those in the core nebula. We predict temperature variations of not much more than $1000 \mathrm{~K}$, and perhaps a positive outward temperature gradient, depending somewhat on the phase NGC 6543 is in.
Our results show that the rings are a transient phenomenon, persisting not much longer than $\sim 3000$ years after photoionization. This seems to be in line with the observed fact that most rings are found in their neutral state around proto-PNe or young PNe (e.g. NGC 7027). In this view the ionization in NGC 6543 is rather recent. In Hb 5 it is not known whether the rings are seen in scattered or intrinsic emission.

Another prediction from our models is that one will not find rings in so-called "attached haloes" or shells around PNe, since these are thought to have been produced by the action of a D-type ionization front (Marten \& Schönberner 1991; Mellema 1994), which as we saw, erases the density variations quite efficiently.

\subsection{Origin of the rings}

To produce our models we started with density and velocity variations produced through the mechanism suggested by Simis et al. (2001), which we slightly modified by taking a lower velocity, and scaling the density to obtain a certain average mass loss rate. Our simulations showed that for the structure during the PN phase, the initial velocity fluctuations are irrelevant, and so the important property is the nature of the density fluctuations. Studying the results of a number of simulations, we found that the key property is the amplitude of the density variations. At the start of the post-AGB phase, these have to be roughly one order of magnitude. Only then ionization leads to large enough pressure fluctuations to result in what could be loosely described as "supersonic turbulence", which helps the longevity of the rings. Without the resulting shock 

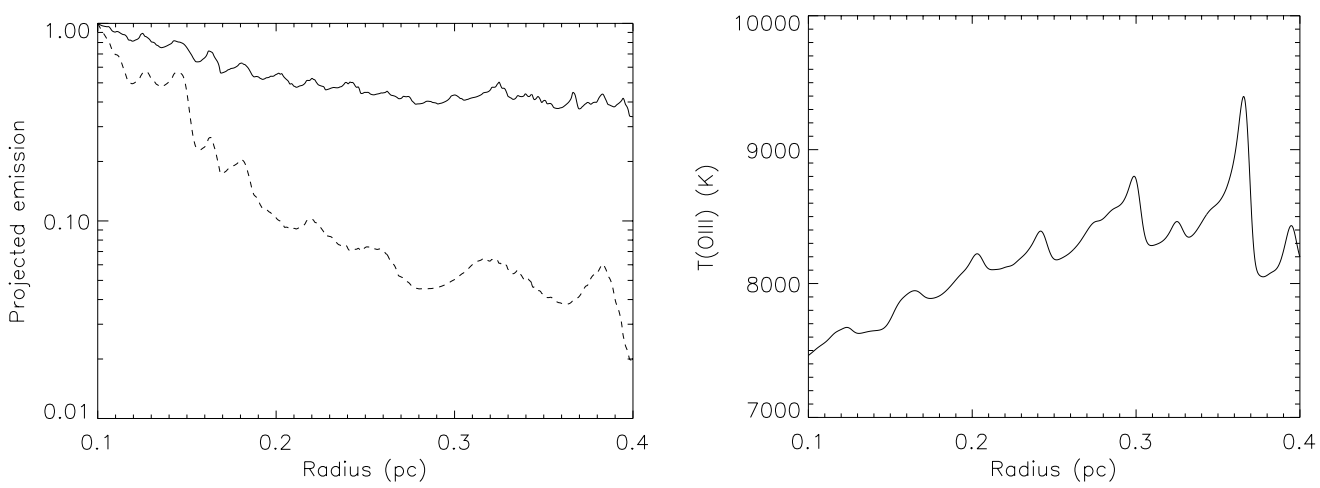

Fig. 10. Left: The projected emission [OIII] (solid) and $\mathrm{H} \alpha$ (dashed) in arbitrary units. Right: Projected temperature. $t=4500$ years.
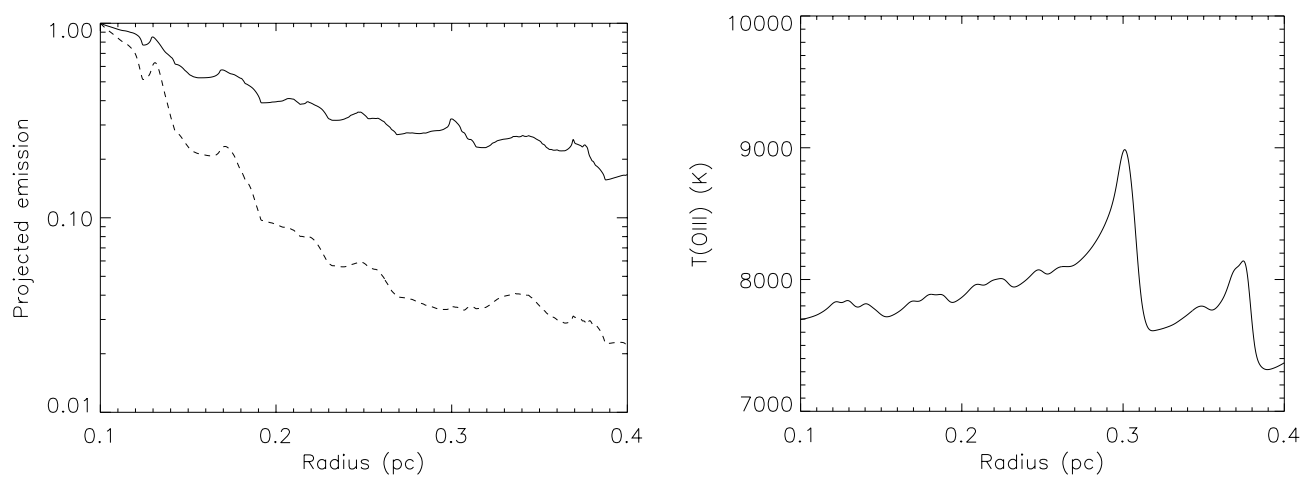

Fig. 11. Left: The projected emission of [OIII] (solid) and $\mathrm{H} \alpha$ (dashed) in arbitrary units. Right: Projected temperature. $t=5500$ years.
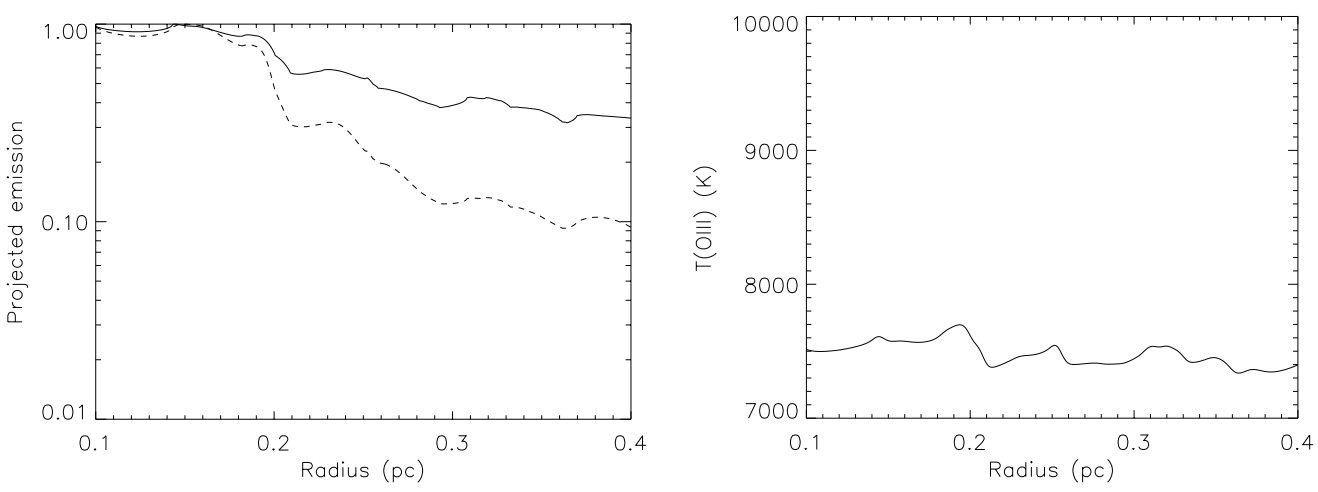

Fig. 12. Left: The projected emission of [OIII] (solid) and $\mathrm{H} \alpha$ (dashed) in arbitrary units. Right: Projected temperature. $t=8000$ years.

waves the rings diffuse away in a few hundred years after ionization.

We would therefore expect that using density variations with similar amplitudes would give more or less the same results. In the model of García-Segura et al. (2001), for reasonable values of the magnetic field, the density initially varies less than in our simulations. However, since these shells are "frozen in", they do not merge, and conserve their density contrast. At the time when the ionization front breaks through, the density contrast is likely to be similar to the one in our simulation. We therefore expect the rings from the MHD model to evolve similarly during the PN phase, and survive for about a few thousand years after ionization, producing broad line profiles as the flow is slowly erasing the rings.

Note that the magnetic pressure is able to support the rings only during the AGB and proto-PN phases. Upon ionization, the magnetic pressure becomes negligible in comparison to the gas pressure, and there is no real difference between the two models. This rather limits the usefulness of the magnetic field, since it is not clear whether it is really needed to maintain the fluctuations up to the time of ionization.

Binary interaction as proposed by Mastrodemos \& Morris (1999) is probably not an explanation for the rings seen in planetary nebulae. The density contrasts they find in their models are much too low to survive for a long time after being ionized, and in fact already seem to be diffusing within a couple of orbital periods.

\section{Conclusions}

We numerically followed the evolution of AGB mass loss fluctuations during the post-AGB phase, paying special attention to 

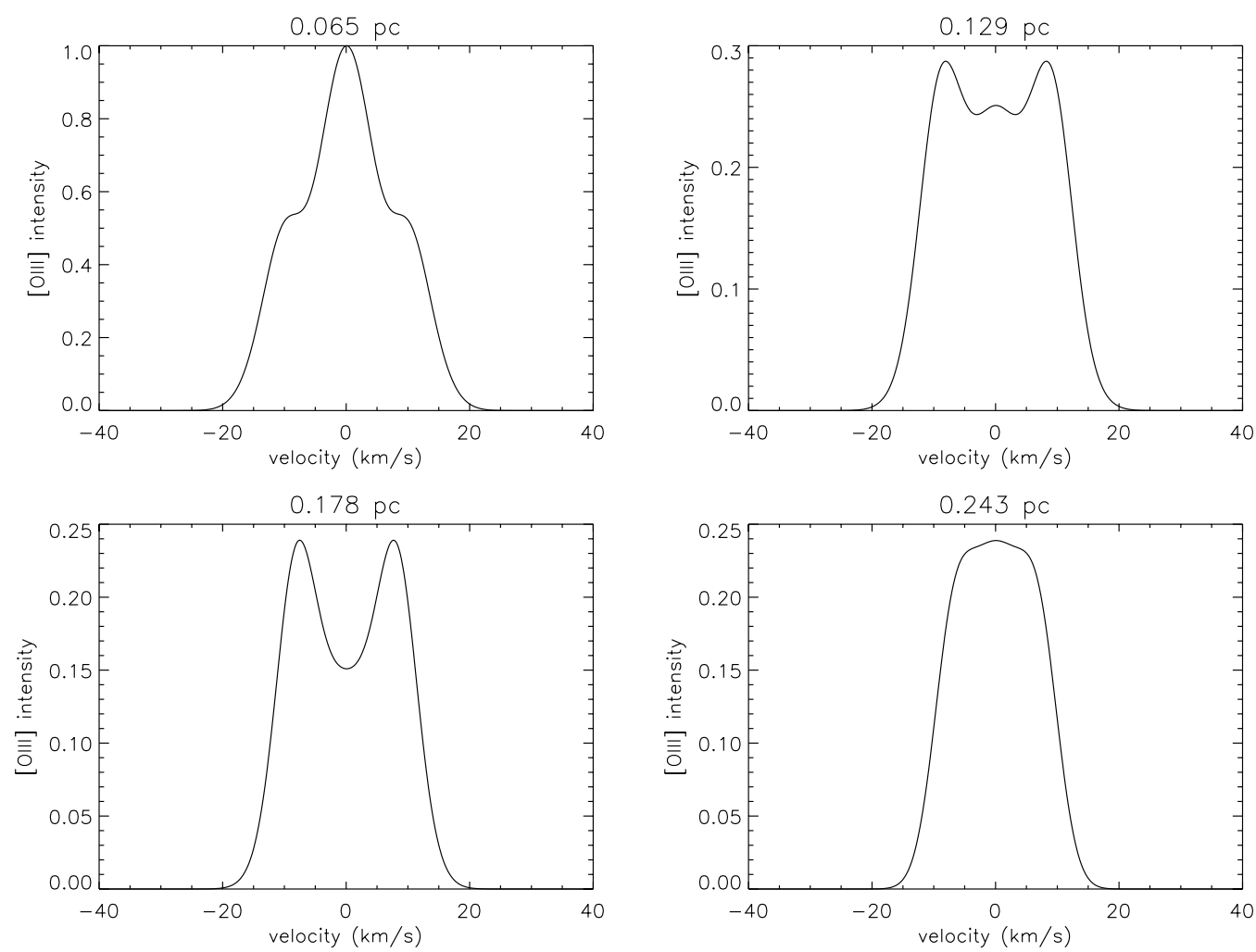

Fig. 13. Lineprofiles for $[\mathrm{OIII}]$ at several distances from the star. The lines are convolved with a Gaussian of $6 \mathrm{~km} \mathrm{~s}^{-1} \mathrm{FWHM}$.

the effects ionization has. We find that using mass loss fluctuations as produced by the mechanism described by Simis et al. (2001) easily survive up to the time ionization sets in for stars of ZAMS mass of $1 M_{\odot}$ or larger. Upon ionization, the pressure fluctuations lead to shocks, which raises the average absolute velocity of the rings, and locally raises the temperature. After a couple of thousand years the rings will have mostly disappeared.

The ionized rings have the following observational characteristics:

- the rings are more pronounced and can be seen further out in $[\mathrm{O}$ III] then in $\mathrm{H} \alpha$;

- only slightly higher electron temperature $( \pm 1000 \mathrm{~K})$ than the rest of the PN, as measured by the [O III] line ratios;

- broad line profiles due to the turbulent velocity field and the many overlapping shells.

We therefore predict that the electron temperature from accurate line ratios of ionized rings will show only a small difference with the electron temperature of the core nebula, showing the result from Hyung et al. (2001) to be spurious. We also predict that high signal-to-noise spectroscopy may reveal nongaussian line shapes in the ring region. Lastly, according to our description, rings will only be found in either neutral or recently ionized haloes.

We found this type of evolution to be quite general, provided that the density fluctuations at the time of ionization are larger than a factor of 10 . For lower values the rings diffuse away through gentle sound waves.

Using this requirement the wide binary model as published by Mastrodemos \& Morris (1999) is disqualified as an explanation for the rings in ionized PNe. The MHD model of García-Segura et al. (2001) and the dust-driven wind instability from Simis et al. (2001) both fulfill the requirements. However, there is some dispute about the choice of period for the magnetic cycle of AGB stars, making the dust-driven wind instability model (with no imposed period) the more likely explanation.

Acknowledgements. The research of GM has been made possible by a fellowship of the Royal Netherlands Academy of Arts and Sciences.

\section{References}

Balick, B., Wilson, J., \& Hajian, A. R. 2001, AJ, 121, 354

Blöcker, T. 1995, VizieR Online Data Catalog, 329, 90755

Boris, J. P. 1976, NRL Mem. Rep., 3237

Bryce, M., Meaburn, J., Walsh, J. R., \& Clegg, R. E. S. 1992, MNRAS, 254, 477

Castor, J. I., Abbott, D. C., \& Klein, R. I. 1975, ApJ, 195, 157

Corradi, R. L. M., Schönberner, D., Steffen, M., \& Perinotto, M. 2003, MNRAS, 340, 417

Dorfi, E. A., \& Höfner, S. 1991, A\&A, 248, 105

Eulderink, F., \& Mellema, G. 1995, A\&AS, 110, 587

Fleischer, A. J., Gauger, A., \& Sedlmayr, E. 1992, A\&A, 266, 321

Frank, A., \& Mellema, G. 1994, A\&A, 289, 937

Gail, H.-P., Keller, R., \& Sedlmayr, E. 1984, A\&A, 133, 320

Gail, H.-P., \& Sedlmayr, E. 1988, A\&A, 206, 153

García-Segura, G., López, J., \& Franco, J. 2001, ApJ, 560, 928

Hrivnak, B. J., Kwok, S., \& Su, K. Y. L. 2001, AJ, 121, 2775

Hyung, S., Mellema, G., Lee, S.-J., \& Kim, H. 2001, A\&A, 378, 587 
Icke, V. 1991, A\&A, 251, 369

Kudritzki, R. P., Pauldrach, A., Puls, J., \& Abbott, D. C. 1989, A\&A, 219, 205

Kwok, S., Su, K. Y. L., \& Hrivnak, B. J. 1998, ApJ, 501, L117

Lamers, H. J. G. L. M., \& Cassinelli, J. P. 1999, Introduction to stellar winds (New York: Cambridge University Press), ISBN 0521593980

Lucy, L. B. 1976, ApJ, 205, 482

Marten, H., \& Schönberner, D. 1991, A\&A, 248, 590

Mastrodemos, N., \& Morris, M. 1999, ApJ, 523, 357

Mauron, N., \& Huggins, P. J. 2000, A\&A, 359, 707

Mellema, G. 1994, A\&A, 290, 915

Mellema, G., \& Lundqvist, P. 2002, A\&A, 394, 901

Mellema, G., Raga, A. C., Canto, J., et al. 1998, A\&A, 331, 335
Osterbrock, D. E. 1989, Astrophysics of gaseous nebulae and active galactic nuclei (University Science Books), 1989

Roe, P. 1981, J. Comp. Phys., 43, 357

Sahai, R., Trauger, J. T., Watson, A. M., et al. 1998, ApJ, 493, 301

Schönberner, D. 1983, ApJ, 272, 708

Simis, Y. J. W., Dominik, C., \& Icke, V. 2003, A\&A, submitted

Simis, Y. J. W., Icke, V., \& Dominik, C. 2001, A\&A, 371, 205

Su, K. Y. L., Volk, K., Kwok, S., \& Hrivnak, B. J. 1998, ApJ, 508, 744

Terzian, Y., \& Hajian, A. R. 2000, in Asymmetrical Planetary Nebulae II: From Origins to Microstructures, ASP Conf. Ser., 199, 33

Winters, J. M., Fleischer, A. J., Gauger, A., \& Sedlmayr, E. 1994, A\&A, 290, 623 